\title{
Longitudinal Association between Late-Life Depression (LLD) and Frailty: Findings from a Prospective Cohort Study (MiMiCS-FRAIL)
}

\author{
M.K. Borges ${ }^{1,2,3}$, C.V. Romanini ${ }^{3}$ N.A. Lima ${ }^{3}$, M. Petrella, D.L. da Costa ${ }^{3}$, V.N. An ${ }^{3}$, B.N. Aguirre ${ }^{3}$, \\ J.R. Galdeano ${ }^{3}$, I.C.Fernandes ${ }^{3}$, J.F. Cecato ${ }^{3}$, E.C. Robello ${ }^{3}$, R.C. Oude Voshaar ${ }^{4}$, I. Aprahamian ${ }^{1,3,4}$
}

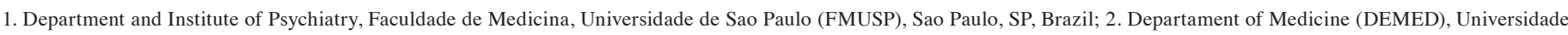

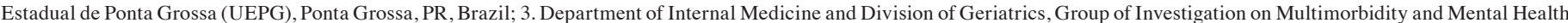

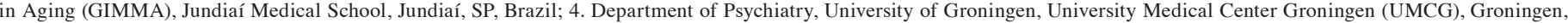
The Netherlands.

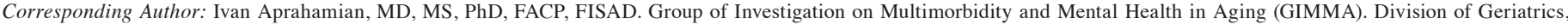
Department of Internal Medicine, Jundiaí Medical School.250 Francisco Telles st. ZIP 13202-550. Jundiaí. Brazil; E-mail: ivan.aprahamian@gmail.com. Tweeter: @IAprahamian

\begin{abstract}
OBJECTIVES: The aim of the present study was to investigate whether late-life depression (LLD) is associated with incident frailty over time.

DESIGN: Prospective cohort study, one-year follow-up.

SETTING: Geriatric outpatient clinic, Southwestern of Brazil.

PARTICIPANTS: 181 follow-up participants aged 60 years or over.

MEASUREMENTS: Depressive disorders were classified as Major Depressive disorder (MDD) or Subthreshold Depression (STD) according to DSM-5 criteria. Depressive symptoms were assessed with validated versions of 15-item Geriatric Depression Scale (GDS15) and 9-item Patient Health Questionnaire (PHQ-9). We performed binary logistic regressions to estimate the odds ratio (OR) for frailty in LLD adjusting for multiple confounders. Participants who were frail at baseline were excluded from the analyses according to measures of frailty (FRAIL questionnaire and 36-item Frailty Index, FI-36). We also estimated the risk ratio or relative risk (RR) and the risk difference (RD) for incident frailty.

RESULTS: We observed a 2 to 4-fold increased risk for incident frailty among participants with LLD. The presence of a depressive disorder was significantly associated with the onset of frailty (adjusted OR for FRAIL and FI-36: 3.07 [95\% CI $=1.03-9.17]$ and 3.76 [95\% CI = 1.09 - 12.97], respectively. Notably, the risk for frailty due to LLD was significantly higher with the FI-36 compared to the FRAIL (RR: 3.03 versus 2.23 ). RD was of $17.3 \%$ and $12.7 \%$ with the FRAIL and the FI-36, respectively.

CONCLUSION: Our data support the association between LLD and incident frailty over one year among geriatric outpatients, reinforcing longitudinal evidence from population-based studies.
\end{abstract}

Key words: Depression, frailty, aged, morbidity, prognosis, geriatric psychiatry.

\section{Introduction}

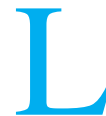

ate-life depression (LLD) accounts for the most common mental illness among older adults in clinical practice $(1,2)$ with a pooled prevalence rate of $7.2 \%$ for a depressive disorder and $17.1 \%$ for clinically relevant depressive symptoms (3). Moreover, the prevalence of LLD tends to be higher in a medical setting among patients compared to community-dwelling older adults $(4,5)$. Clinically, LLD differs from depression in younger adults by having higher rates of recurrence, more somatic comorbidities, more cognitive and functional impairment, and finally by its association with frailty $(6,7)$.

Frailty is a geriatric syndrome defined as an increased state of organic vulnerability and loss of resistance to stressors due to a reduced reserve in several physiological systems (8). Frailty is mostly operationalized by meeting at least three out of five criteria of the physical phenotype or with a score above the cut-off of the Frailty Index (FI) a multidimensional measure reflecting the proportion of at least 30 potential health deficits (9). A recent systematic review and meta-analysis showed that the prevalence of physical frailty is $12 \%(95 \% \mathrm{CI}=11-13 \%)$ for frailty phenotype and up to $24 \%(95 \% \mathrm{CI}=22-26 \%)$ when based on the FI (10). The prevalence of frailty in low to middle income countries is higher compared to the more developed countries and is estimated at $17.4 \%(95 \% \mathrm{CI}=14.4-20.7 \%)$ (11).

LLD and frailty are associated with adverse health outcomes $(12,13)$. Both conditions predisposes to a worse quality of life, burden of other diseases, greater cognitive decline, and higher disability rates (14-17). Three to four out of ten older adults with depression are frail, which may contribute to the increased mortality rates found among depressed individuals $(13,18,19)$. Two meta-analyses have found that LLD and frailty have a reciprocal association $(18,20)$. Nonetheless, studies included in these meta-analyses showed a high degree of heterogeneity $(18,20)$. This may be explained by the many operationalisation of frailty (in particular) which more or less overlap with the criteria for a depressive disorder $(21,22)$ as well as the use of self-report screening tests to evaluate LLD (20). Four longitudinal studies showed a four-fold incident frailty among depressed seniors (18). However, these studies involved community-dwelling older adults and included only physical dimensions of frailty.

Evidence from clinical samples is scarce and most are cross-sectional involving either psychiatric outpatients (23, 24), renal transplant patients (25), geriatric inpatients (26) or are longitudinal studies in psychogeriatric clinics $(19,27$, 28). Older adults referred to geriatric outpatient clinics are 
potentially more complex due to higher rates of multimorbidity, frailty and depression compared to community samples (29). The only longitudinal study in a sample with geriatric outpatients showed a 2.8 odds of incident frailty among depressed older adults treated with selective serotonin reuptake inhibitors monotherapy (30).

The present study has been designed to test whether depression is associated with an increase of frailty over time using the FI in addition to the self-report FRAIL questionnaire. The FI has a higher predictive ability of adverse events than other frailty measurements in both hospital and community settings $(26,31)$. Although the FI can be easily calculated based on data routinely collected in digital health records, it has been rarely used for assessing frailty in association with LLD and thus far only by cross-sectional analyses $(32,33)$. The aim of the present study was to investigate whether late-life depression (LLD) is associated with incident frailty.

\section{Methods}

\section{Study design, participants and procedures}

The Multimorbidity and Mental health Cohort Study in Frailty and Aging (MiMiCS-FRAIL) is an ongoing prospective cohort study aimed to investigate the interplay between LLD, frailty and inflammation started in January 2018 (34). Participants of the cohort are eligible outpatients of a university-based interdisciplinary geriatrics clinic in Southwestern of Brazil (city of Jundiaí, State of São Paulo). The present study included data from the first wave with a 1-year follow-up.

Eligible participants of the MiMiCS-FRAIL are all new referrals to the clinic (from general practitioners or patient's direct access) aged 60 years or over, with regular clinical appointment and follow-up (at least one visit to the clinic every 12 months). Exclusion criteria are: (1) refusal to participate in the research; (2) dementia; (3) bipolar disorder; (4) psychotic disorder; (5) delirium or hospitalization in the last 30 days; (6) electroconvulsive therapy (ECT) treatment; (7) wheelchair dependent; (8) severe sensory impairment; (9) severe motor impairment due to stroke; (10) unstable clinical condition (e.g., decompensated heart failure, current infection); (11) terminal illness.

All included participants had a baseline assessment with a comprehensive geriatric assessment (CGA) protocol including a structured diagnostic interview, a complete physical and psychiatric evaluation and validated self-report questionnaires with a team of geriatricians, psychiatrists and physical therapists (32-34). Subsequently, patients are routinely followed-up every 12 months (at least) with the CGA.

\section{Ethical considerations}

The MiMiCS-FRAIL follows the ethical standards established by the Brazilian National Council of Health and is conducted in accordance with the human's rights and recommendations stipulated by the Helsinki Convention. Local and national ethical committee (University of São Paulo and Jundiai Medical School) approved this study. All participants signed an informed consent.

\section{Measurements}

\section{Depression}

LLD was initially evaluated by a geriatrician and posteriorly by a geriatric psychiatrist who confirmed the diagnosis of a depressive disorder following the mood section of the Structured Clinical Interview for DSM-5 disorders Clinical Version (SCID-5-CV). Depressive disorders were classified as either Major Depressive disorder (MDD) or Subthreshold Depression (STD) defined as «another specified depressive disorder» according to DSM-5 criteria (35). All participants were clinically evaluated for the exclusion of bipolar illness as well as to depression syndrome secondary to a medical condition by two geriatric psychiatry specialists. All diagnosis were confirmed in a consensus meeting of the clinical research team.

Validated versions of the 15-item Geriatric Depression Scale (GDS-15) (36) and the Patient Health Questionnaire (PHQ) 9-item version $(37,38)$ were used to measure depressive symptoms and depression severity $(32,33)$. Mild MDD corresponded to a score between 5 to 9 points, moderate from 10 to 14 , moderately severe from 15 to 19 , and severe from 20 to 27 points in PHQ-9 (6).

\section{Frailty}

Frailty was assessed in both baseline and followup according to two validated instruments, i.e. the FRAIL questionnaire (39-41) and the FI (42-44). The FRAIL questionnaire is a self-report screening questionnaire based on components of the Fried frailty phenotype (8) combined with the presence of multimorbidity in Frailty Index models (43). The FRAIL assesses the presence of fatigue, muscle resistance, ambulation, multimorbidity, and weight loss based on the following questions: (1) Fatigue: the answers "all the time" or "most of the time" to the question "How much of the time during the past 4 weeks did you feel tired?"; (2) Resistance: "yes" to the question "By yourself and not using aids, do you have any difficulty walking up 10 steps without resting?"; (3) Ambulation: "yes" to the question "By yourself and not using aids, do you have any difficulty walking several hundred yards?"; (4) Illness: presence of five or more illnesses out of 11; and (5) Loss of weight: respondents with a weight loss $\geq 5 \%$ of their total weight within one year. One point is given to an affirmative answer yielding a total score between 0 and $5 ; \geq 3$ points represents frailty.

- A previously validated 36-item Frailty Index (FI-36) was used (45). The FI-36 accounts for the proportion of accumulated deficits derived from a count of 36 symptoms, signs, laboratorial exams, conditions and disabilities across different health domains (ranging from 0 to 1 ). The index is achieved by the sum of present variables divided by 
Table 1. Socio-demographic and clinical characteristics according to depression status $(\mathrm{n}=181)$

\begin{tabular}{|c|c|c|c|c|c|}
\hline \multirow[t]{2}{*}{ Characteristics } & & \multicolumn{3}{|c|}{ Depression status } & \multirow[b]{2}{*}{$\mathbf{P}$} \\
\hline & & Non-depressed (n=92) & STD $(n=43)$ & $\operatorname{MDD}(n=46)$ & \\
\hline \multicolumn{6}{|l|}{ Demographics: } \\
\hline Female & $\mathrm{n}(\%)$ & $47(51.1)$ & $26(60.5)$ & $27(58.7)$ & 0.512 \\
\hline Education (years) & Mean (SD) & $5.6(4.5)$ & $4.6(4.5)$ & $5.5(4.8)$ & 0.498 \\
\hline \multicolumn{6}{|l|}{ Clinical characteristics: } \\
\hline BMI (kg/m2) & Mean (SD) & $27.7(4.5)$ & $26.7(4.3)$ & $27.8(5.7)$ & 0.422 \\
\hline Cognitive functioning (10-CS) & Mean (SD) & $7.3(2.5)$ & $6.7(2.2)$ & $6.1(2.6)$ & 0.021 \\
\hline Multimorbidity & $\mathrm{n}(\%)$ & $19(20.7)$ & $9(20.9)$ & $16(34.8)$ & 0.159 \\
\hline Polypharmacy & $\mathrm{n}(\%)$ & $53(57.6)$ & $31(72.1)$ & $34(73.9)$ & 0.092 \\
\hline \multicolumn{6}{|l|}{ Depression: } \\
\hline \multicolumn{6}{|l|}{ Frailty: } \\
\hline Screening (FRAIL) & $\mathrm{n}(\%)$ & $14(15.2)$ & $20(46.5)$ & $34(73.9)$ & $<0.001$ \\
\hline Frailty Index (FI-36) & Mean (SD) & $0.12(0.07)$ & $0.17(0.06)$ & $0.21(0.09)$ & $<0.001$ \\
\hline
\end{tabular}

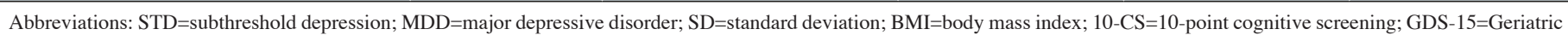

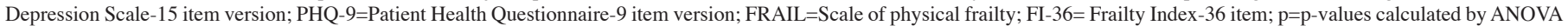

(in case mean (SD) are presented) or $\chi 2$ test (in case $\mathrm{n}(\%)$ are presented). Bold values signifies $\mathrm{p}<.05$

total variables included. The FI is a stronger predictor for adverse health outcome such as death than the presence of its individual components or multimorbidity $(42,46)$. The following 36 health deficits were included: anaemia, arthritis, cognitive impairment, visual impairment, diabetes, dyspnea, chronic renal disease, sleep disorder, peripheral vascular diseases, urinary tract disorders, thyroid disease, respiratory disease, cerebrovascular disease, ischemic heart disease, atrial fibrillation, fracture, hypertension, syncope, heart failure, urinary incontinence, disability, care dependency, osteoporosis, falls, parkinsonism and related disorders, loss of appetite or anorexia, polypharmacy, foot disorders, mobility problems, obesity, hearing loss, valvulopathy, dizziness, social vulnerability, pressure ulcers, peptic ulcers. Frailty was considered with a FI-36 of $\geq 0.25$ $(43,44)$.

\section{Covariates}

Age, sex, years of education, body mass index, cognitive performance (according to the 10-Cognitive Screener test) (47), multimorbidity ( $\geq 2$ chronic diseases), and polypharmacy ( $\geq 5$ medications in current use) were included as covariates. These variables were chosen due to their previous association with both depression and frailty in the literature $(9,23,48-50)$.

\section{Statistical analysis}

Descriptive statistics (proportion or mean with standard deviation) were presented to characterize the sample. All continuous variables showed a normal distribution based on the histogram and Shapiro-Wilk test. One-way ANOVA (continuous variables) or Chi-square test $(\chi 2)$ (categorical variables) were applied to compare patients with either MDD, STD or no depression.

Of the follow-up participants, those who were frail at baseline and with missing data were excluded from the analyses, according to different definitions of frailty (the FRAIL questionnaire and the FI-36, respectively). Among non-frail participants at baseline, we performed binary logistic regressions to estimate the odds ratio (OR) for frailty (dependent variable) in participants with MDD or STD versus no-depression at baseline (independent variable). We also estimated the risk ratio or relative risk (RR) and the risk difference (RD) for incident frailty.

Goodness of fit was tested through Akaike Information Criterion (AIC) values, Hosmer-Lemeshow test and R2 (Cox-Snell). Multicollinearity problems were checked using the variance inflation factor. All regression models were adjusted for multiple confounders (described as covariates) and conducted for both the FRAIL questionnaire as well as the FI separately. P-values lower than 0.05 were considered statistically significant. Data were analysed using Statistical Package of the Social Sciences (SPSS), version 25.0.

\section{Results}

\section{Characteristics of the sample}

Of the 315 patients willing to participate at baseline, 134 met the exclusion criteria or lost to follow-up (see Figure 1), i.e., 
Figure 1. Flowchart of the samples (according to different definitions of frailty)

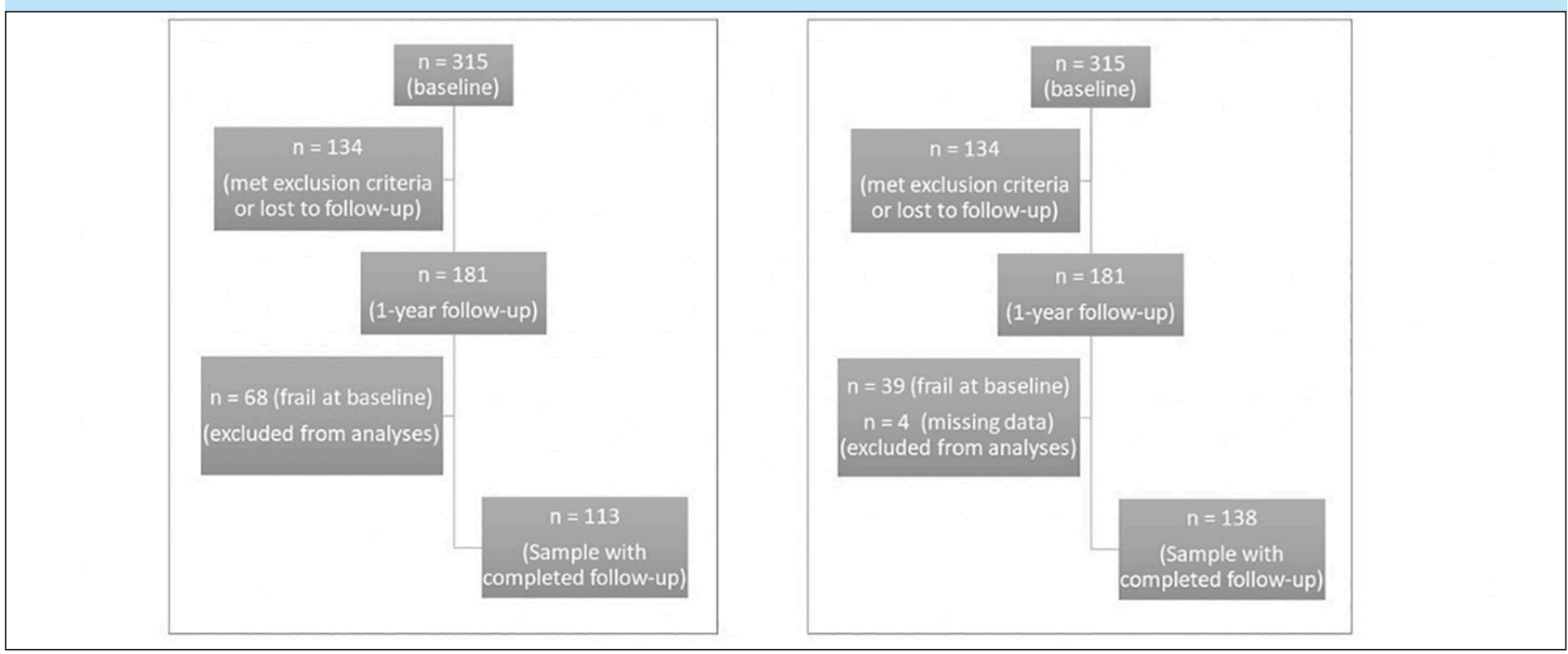

Figure 2. Flowchart of prospective samples

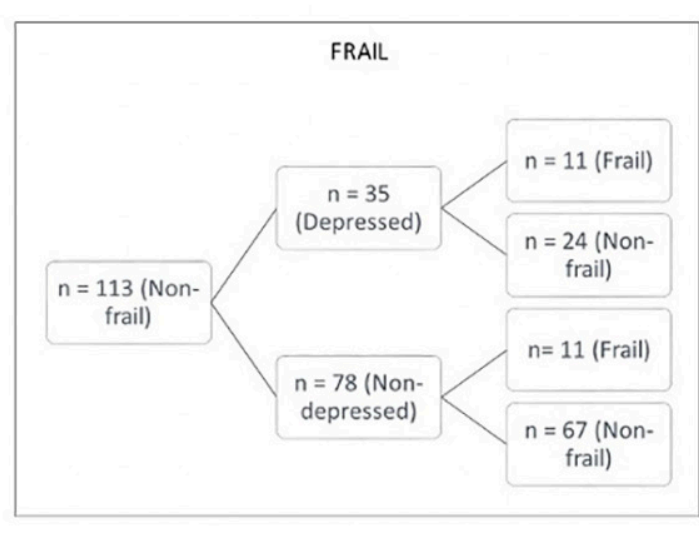

59 participants dropped out (withdraw of consent due to fear of COVID-19), 49 were too ill to participate (32 hospitalization in the last 30 days; 10 dementia cases, 3 severe motor impairment due to stoke; 2 terminal illness; 1 severe sensory impairment; 1 wheelchair dependent) and 26 deceased. The mean age of the 181 participants was 73.2 years, and $55.2 \%$ were women. Furthermore, 46/181 (25.4\%) participants had major depressive disorder (MDD) and 43/181 (23.8\%) subthreshold depression. Table 1 presents follow-up characteristics, stratified by depression status.

\section{Prospective associations between depression and frailty at one-year follow-up}

Of the 181 follow-up participants, 113 were non-frail at baseline according to the FRAIL questionnaire. Figure 2 shows the proportion of frail and non-frail within the depressed group compared to non-depressed one. The incidence rate of frailty in the depressed group versus non-depressed was (11/35) $31.4 \%$ versus $(11 / 78) 14.1 \%$, respectively $(\chi 2=4.62, \mathrm{df}=1$, $\mathrm{p}=0.031)$. Therefore, the RD was $17.3 \%$ and the RR was 2.23

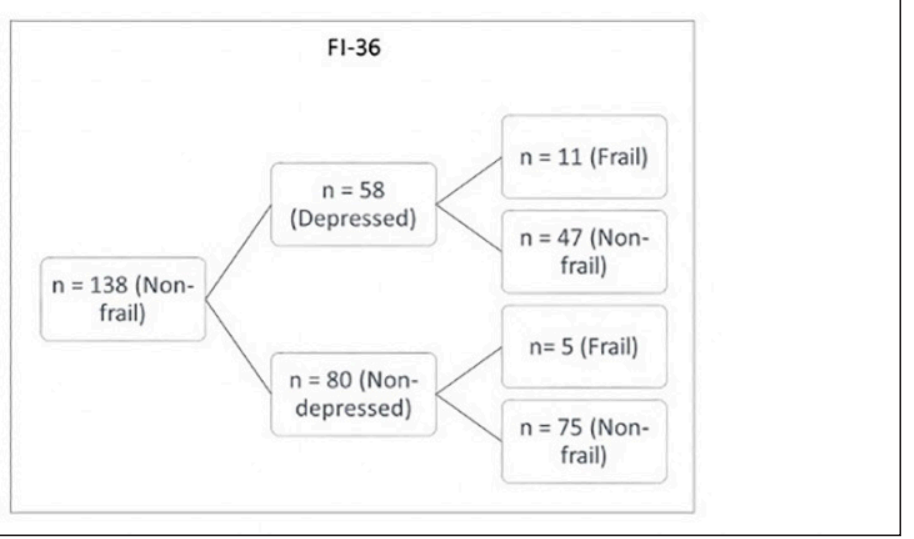

[95\% CI: $1.07-4.64]$.

Of the 181 follow-up participants, 138 were non-frail at baseline regarding FI-36 (see Figure 2). The incidence rate of frailty in the depressed group versus non-depressed was (11/58) $19 \%$ versus $(5 / 80) 6.3 \%$, respectively $(\chi 2=5.30, \mathrm{df}=1, \mathrm{p}=$ $0.021)$. Furthermore, the RD was $12.7 \%$ and the RR was 3.03 [95\% CI $=1.11-8.26]$.

Table 2 shows the odds ratios for incident frailty in either patients with STD or MDD. Based on the FRAIL questionnaire, only MDD was significantly associated with the onset of frailty at follow-up in the fully adjusted analyses. Based on the FI-36, neither STD nor MDD was significantly associated with the onset of frailty at follow-up. Nonetheless, since the statistical power was rather low, we post-hoc tested whether the presence of any depressive disorder (either STD or MDD) was associated with the onset of frailty. This analyses showed that the presence of a depressive disorder was significantly associated with the onset of frailty as measured with the FRAIL questionnaire (unadjusted: $\mathrm{OR}=2.79[95 \% \mathrm{CI}=1.07-7.27], \mathrm{p}=0.036$; adjusted: $\mathrm{OR}=3.07[95 \% \mathrm{CI}=1.03-9.17], \mathrm{p}=0.044)$ as well as with the FI-36 (unadjusted: $\mathrm{OR}=3.51[95 \% \mathrm{CI}=1.15$ $-10.74], \mathrm{p}=0.028$; adjusted: $\mathrm{OR}=3.76[95 \% \mathrm{CI}=1.09-$ 
Table 2. Prospective associations between baseline depression (independent variable) and different definitions of frailty at oneyear follow-up (dependent variable) by logistic regression

\begin{tabular}{|c|c|c|c|c|c|c|}
\hline & \multicolumn{3}{|c|}{ FRAIL $(n=113)$} & \multicolumn{3}{|c|}{ FI-36 $(n=138)$} \\
\hline & OR & $95 \% \mathrm{CI}$ & $\mathbf{p}$ & OR & $95 \% \mathrm{CI}$ & p \\
\hline \multicolumn{7}{|l|}{ Depressive disorders (DSM-5): } \\
\hline \multicolumn{7}{|l|}{ Model 1: } \\
\hline Subthreshold depression (STD) & 2.15 & $(0.69-6.64)$ & 0.184 & 3.75 & $(1.10-12.79)$ & 0.035 \\
\hline Major depressive disorder (MDD) & 4.35 & $(1.17-16.17)$ & 0.028 & 3.16 & $(0.77-12.91)$ & 0.109 \\
\hline \multicolumn{7}{|l|}{ Model 2*: } \\
\hline Subthreshold depression (STD) & 2.29 & $(0.64-8.26)$ & 0.204 & 3.68 & $(0.95-14.30)$ & 0.060 \\
\hline Major depressive disorder (MDD) & 4.80 & $(1.12-20.65)$ & 0.035 & 3.90 & $(0.80-18.93)$ & 0.091 \\
\hline \multicolumn{7}{|l|}{ Depressive symptoms: } \\
\hline \multicolumn{7}{|l|}{ Model 1: } \\
\hline Depression severity (PHQ-9) & 1.23 & $(1.10-1.36)$ & $<0.001$ & 1.14 & $(1.05-1.24)$ & 0.002 \\
\hline Depressive symptoms (GDS-15) & 1.39 & $(1.15-1.68)$ & 0.001 & 1.25 & $(1.06-1.47)$ & 0.007 \\
\hline \multicolumn{7}{|l|}{ Model 2*: } \\
\hline Depression severity (PHQ-9) & 1.27 & $(1.12-1.45)$ & $<0.001$ & 1.12 & $(1.02-1.24)$ & 0.023 \\
\hline Depressive symptoms (GDS-15) & 1.39 & $(1.14-1.71)$ & 0.001 & 1.25 & $(1.02-1.53)$ & 0.031 \\
\hline
\end{tabular}

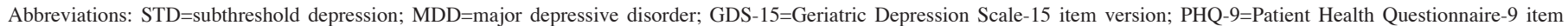

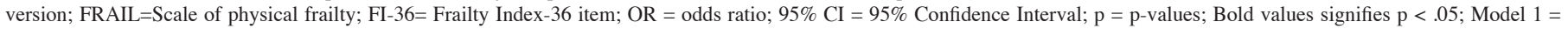
unadjusted model; Model 2 = fully adjusted model; * Adjusted for age, sex, education, BMI, cognition, multimorbidity and polypharmacy.

Figure 3. Odds ratios for frailty (according to the FRAIL and the FI-36)

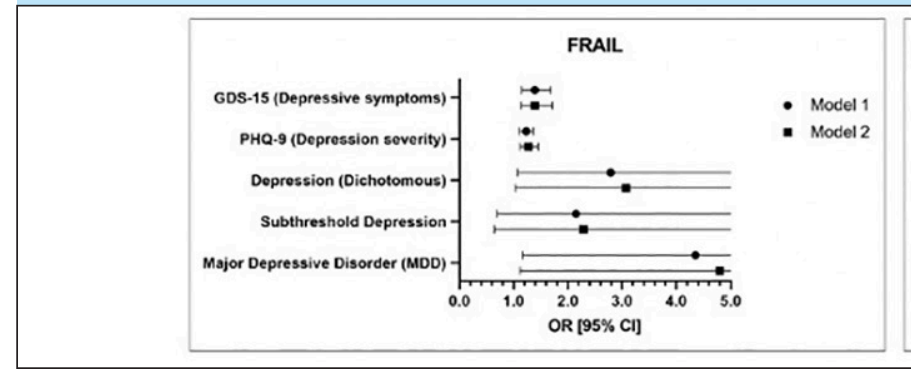

12.97], $\mathrm{p}=0.036$ ). Both measures of depressive symptoms (measured by PHQ-9 and GDS-15) were also associated with a higher odds ratio for frailty according to the FRAIL and FI-36 (Table 2 and Figure 3).

\section{Discussion}

\section{Main findings}

In summary, this prospective cohort study found a 2 to 4 -fold increased risk for incident frailty among non-frail participants with depression (mainly depressive disorders) compared to nondepressed patients. Regarding the hypothesis of this study, our data support the association between LLD and incident frailty over a one-year follow-up among geriatric outpatients. Findings were rather robust regarding the measurement of either frailty or depression.

The incidence rates of frailty in the depressed group were higher than the non-depressed one, and are in line with previous studies $(30,48,49,51,52)$. The incidence of frailty varies

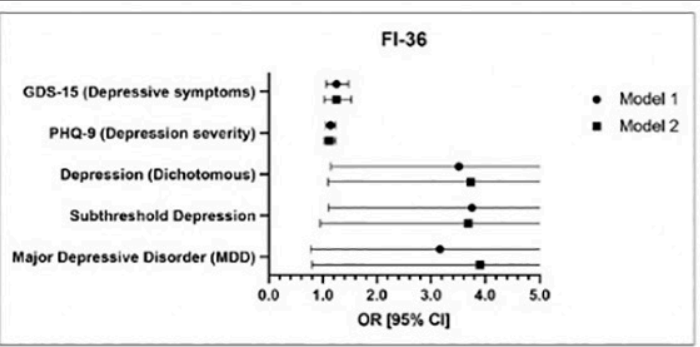

depending on the population being assessed and the diagnostic criteria used. We demonstrated that both frailty measures are roughly similar with respect to the onset of frailty. Four studies found an incident frailty from $9 \%(30,52)$ to $15 \%(48,49)$ when compared to $19 \%$ in our sample with the FI. In the study conducted by Paulson and colleagues (51), the incidence of frailty was of $31.8 \%$, a similar rate found in the current study (31.4\%) with the FRAIL questionnaire.

A recent systematic review and meta-analysis demonstrated that the prospective relationship between depressive symptoms and incident frailty was robust (20). This review concluded that depressed older adults are 3 times more at risk to develop frailty than those without depression (20). In line with this study, we found a RR of 2 to 3 for incident frailty depending on the criteria used to define frailty. Notably, the risk for frailty due to depression was significantly higher when used FI compared to FRAIL (RR for FI and FRAIL: 3.03 and 2.23, respectively).

On the other hand, frailty and depression may share a common vulnerability to the same stressors, which could result in an overlap of both conditions $(22,18)$. Even though this hypothesis was recently confirmed (53), it contradicts 
evidence-based literature review which has demonstrated a uni- or bi-directional associations between both constructs $(18,20,21)$. For instance, a population-based cohort study, Rugao Longevity and Aging Study (RuLAS), showed that depressive symptoms (measured by the GDS-15) were related to the emergence of frailty over a 3-years follow-up $(\mathrm{OR}=$ $2.79[95 \% \mathrm{CI}=1,09-7,10])$, after adjusting for covariates (52). Chu and colleagues (54) also confirmed that frailty (measured by Fried frailty phenotype) was associated with depressive symptoms (measured by GDS) after longitudinal analyses (OR $=2.12,95 \% \mathrm{CI}=1.17-3.83)$, considering the adjusted models . As previously mentioned $(52,54)$, the same cohort study investigated the relationship between depressive symptoms and frailty reciprocally, which reinforces the bidirectional association between both conditions.

However, the current study extends to the findings of these studies that depressive disorders (mainly MDD) also predicts the onset of frailty. The core symptoms of MDD according to the DSM-5 describe a specific depression syndrome (6) so adopting the diagnostic criteria for depression adds the credibility to the methodology used in this study. Depression screening instruments (i.e. GDS-15 and PHQ-9) do not immediately lead to a diagnosis but can accurately identify patients who are at risk. In addition, our findings can contribute with the assessment of depressive symptoms that seem to yield similar and good results $(32,33)$. These results could be explained by findings that depressive symptoms were associated with an increased risk for frailty $(\mathrm{OR}=2.20$, [95\% CI $=1.88-2.57]$ ) over 3-years follow-up (48). The same cohort study has shown that depression severity was a risk factor for frailty (severe symptoms, OR $=2.19[95 \% \mathrm{CI}=$ 1.86-2.59]; moderate symptoms, OR $=1.31$ [95\% CI $=1.14$ 1.50]) (49). Paulson and colleagues (51) found a 2.8 -fold for the incident frailty, considering the profile of greater burden of cerebrovascular disease and more severity of depressive symptoms, among women with vascular depression.

Due to a lack of relevant data, the current study provides limited information on potential underlying factors and biological plausibility. Some previous studies assessed the pathophysiology and causation of frailty in depressed older adults. A cross-sectional study from the NESDO evaluated the association between physical frailty and low-grade inflammation in LLD assessing three inflammatory markers, namely, C-reactive protein (CRP), interleukin-6 (IL-6) and neutrophil gelatinase-associated lipocalin (NGAL) (55). Among the most relevant findings, frailty or severity of depressive symptoms were not associated with higher levels of inflammatory markers. In addition, the findings of the Health Aging and Body Composition Study (HABCS) reinforces these analyses due to lack of evidence between inflammation, depression, slow gait and mortality (56). Inflammation, depression and slow gait define a phenotype at risk of death. Trajectories of inflammation had an independent effect on mortality after accounting for covariates and the effect of trajectories of slow gait on mortality depended on depression status (56). Arts and colleagues (57) investigated the association between leucocyte telomere length (LTL) as molecular marker of aging and the frailty phenotype and whether these associations would be moderated by the presence of a depressive disorder. LTL was related to frailty but with a really small effect on LLD (57). Arts and colleagues (19) showed that frailty and aging related biomarkers (especially CRP and vitamin D) predicts mortality among depressed older patients. Therefore, there is a lack of validity of these results mainly in longitudinal studies that could confirm the pathogenesis and the causation of frailty in LLD.

\section{Strengths and limitations}

To our knowledge, this is the first prospective study to examine LLD and frailty (assessed with the FI and a CGA) in a geriatric outpatient clinic. Thus, this is clinically relevant because depressed geriatric outpatients have an increased risk of becoming frail. In addition, the prevalence of frailty in Brazil is higher in those individuals recruited from health care services when compared to community ones (30\% versus $22 \%$, respectively) (58).

Second, the current study focused on the changes in the FI, because of its robust flexibility and wide reproducibility that does not depend on which deficits are chosen (44). It is crucial to emphasize that both frailty measures were roughly similar regarding the onset of frailty. The FRAIL is a really feasible screening instrument and highly relevant in clinical practice (40). Therefore, the FRAIL questionnaire was used as a measure of comparison with the changes in the FI in order to infer whether our findings would be consistent.

Third, our analyses were based on DSM-5 criteria, in order to distinguish MDD from STD instead of considering only depressive symptoms. STD is generally also seen as an important depressive syndrome in late life (59). Most previous studies have only assessed self-report depressive symptoms in the association with frailty phenotype and this may be confounded with the presence of frailty $(18,20)$.

Finally, the assessment and control of confounders were performed by stratifying the study sample and using fullyadjusted statistical analyses on the association between LLD and frailty. This could explain one possible direction of this association and internally validate our predictive model. Therefore, our main findings represent as not being a result by chance neither of a potential measurement or confounding biases.

Limitations of the current study should be also considered. We are unable to generalize completely our findings to other groups because our patients presented lower levels of schooling and worse cognitive functioning when compared to general population. In addition, our sample size could have resulted in underpowered analyses (some participants dropped out due to fear of COVID-19) and a larger sample might find results more robust. Other studies could replicate and validate these findings to other clinical settings with different basedpopulation samples. 


\section{Conclusions and implications}

According to the hypothesis of this study, it has been confirmed that depressive disorders predicts the changes in frailty. For instance, a depressed older adult becomes increasingly sedentary and socially isolated, resulting in a greater odds of developing physical symptoms of frailty such as weakness, exhaustion and slow gait. Thus, screening for both LLD and frailty is crucial in order to reduce the burden of these clinical conditions and adverse health outcomes in older adults.

Future research should be directed to successful treatment of LLD in frail seniors because of the potential capacity for psychosocial interventions to improve physical-health outcomes. Prince and colleagues (60) have already highlighted: "no health without mental health". As a result, this approach could lead to an increased behavioral and social activation, thereby improving the levels of physical and social activity in these individuals, and, thus, reducing the risk of the onset and progression of frailty. Finally, further studies should address the underlying mechanisms in the relationship between LLD and frailty.

\section{Disclosure statement: No potential conflict of interest was reported by the authors.}

Funding: This study was financed in part by the "Coordenação de Aperfeiçoamento de Pessoal de Nível Superior - Brasil (Capes)" - Finance Code 001. Professor Aprahamian received a national public grant level two from the National Council for Scientific and Technological Development (Ministry of Science, Technology, Innovation and Communications, Brazil).

Ethical standards: This study was conducted according to the ethical guidelines of research with human beings, approved by the local Ethics Committee.

\section{References}

1. Hall CA, Reynolds-III CF. Late-life depression in the primary care setting: challenges, collaborative care, and prevention. Maturitas 2014;79:147-152. doi.org/10.1016/j. maturitas.2014.05.026

2. Taylor WD. Clinical practice. Depression in the elderly. The New England journal of medicine 2014;371:1228-1236. doi.org/10.1056/NEJMcp1402180

3. Luppa, M. et al. Age- and gender-specific prevalence of depression in latest-life-systematic review and meta-analysis. Journal of affective disorders 2012;136:212-221. doi.org/10.1016/j.jad.2010.11.033

4. Alexopoulos GS. Depression in the elderly. Lancet 2005;365:1961-1970. doi. org/10.1016/S0140-6736(05)66665-2

5. Sjöberg L, Karlsson B, Atti AR, Skoog I, Fratiglioni L, Wang HX. Prevalence of depression: Comparisons of different depression definitions in population-based samples of older adults. Journal of affective disorders 2017;221:123-131. doi. org/10.1016/j.jad.2017.06.011

6. McCarron RM, Vanderlip ER, Rado J. Depression. Annals of internal medicine 2016;165: ITC49-ITC64. doi.org/10.7326/AITC201610040

7. Alexopoulos GS. Mechanisms and treatment of late-life depression. Translational psychiatry 2019;9:188. doi.org/10.1038/s41398-019-0514-6

8. Fried LP et al. Frailty in older adults: evidence for a phenotype. The journals of gerontology. Series A, Biological sciences and medical sciences 2001;56:M146-M156. doi.org/10.1093/gerona/56.3.m146

9. Hoogendijk EO, Afilalo J, Ensrud KE, Kowal P, Onder G, Fried LP. Frailty: implications for clinical practice and public health. Lancet 2019;394:1365-1375. doi. org/10.1016/S0140-6736(19)31786-6

10. O'Caoimh R et al. Prevalence of frailty in 62 countries across the world: a systematic review and meta-analysis of population-level studies. Age and ageing 2020; afaa219. Advance online publication. doi.org/10.1093/ageing/afaa219

11. Siriwardhana DD, Hardoon S, Rait G, Weerasinghe MC, Walters KR. Prevalence of frailty and prefrailty among community-dwelling older adults in low-income and middle-income countries: a systematic review and meta-analysis. BMJ open 2018;8(3):e018195. doi.org/10.1136/bmjopen-2017-018195

12. Lohman MC, Mezuk B, Dumenci L. Depression and frailty: concurrent risks for adverse health outcomes. Aging \& mental health, 2017;21:399-408. doi.org/10.1080/1 3607863.2015.1102199
13. Almeida OP, Hankey GJ, Yeap BB, Golledge J, Norman PE, Flicker L. Depression, frailty, and all-cause mortality: a cohort study of men older than 75 years. Journal of the American Medical Directors Association 2015;16:296-300. doi.org/10.1016/j. jamda.2014.10.023

14. Clegg A, Young J, Iliffe S, Rikkert MO, Rockwood K. Frailty in elderly people. Lancet 2013;381:752-762. doi.org/10.1016/S0140-6736(12)62167-9

15. Kojima G, Iliffe S, Jivraj S, Walters K. Association between frailty and quality of life among community-dwelling older people: a systematic review and meta-analysis. Journal of epidemiology and community health 2016;70:716-721. doi.org/10.1136/ jech-2015-206717

16. Nascimento PP, Batistoni SS, Neri AL. Frailty and depressive symptoms in older adults: data from the FIBRA study - UNICAMP. Psicologia: Reflexão e Crítica 2016;29:16. doi.org/10.1186/s41155-016-0033-9

17. Almeida OP, Hankey GJ, Yeap BB, Golledge J, Hill KD, Flicker L. Depression Among Nonfrail Old Men Is Associated With Reduced Physical Function and Functional Capacity After 9 Years Follow-up: The Health in Men Cohort Study. Journal of the American Medical Directors Association 2017;18:65-69. doi.org/10.1016/j. jamda.2016.09.002

18. Soysal $\mathrm{P}$ et al. Relationship between depression and frailty in older adults: A systematic review and meta-analysis. Ageing research reviews 2017;36:78-87. doi. org/10.1016/j.arr.2017.03.005

19. Arts MHL et al. Frailty as a predictor of mortality in late-life depression: a prospective clinical cohort study. J Clin Psychiatry. 2021;82(3):20m13277. doi.org/10.4088/ JCP.20m13277.

20. Chu W, Chang SF, Ho HY, Lin HC. The Relationship Between Depression and Frailty in Community-Dwelling Older People: A Systematic Review and Meta-Analysis of 84,351 Older Adults. Journal of nursing scholarship : an official publication of Sigma Theta Tau International Honor Society of Nursing 2019;51:547-559. doi.org/10.1111/ jnu.12501

21. Mezuk B, Edwards L, Lohman M, Choi M, Lapane K. Depression and frailty in later life: a synthetic review. International journal of geriatric psychiatry 2012;27:879-892. doi.org/10.1002/gps.2807

22. Brown PJ et al. The Depressed Frail Phenotype: The Clinical Manifestation of Increased Biological Aging. The American journal of geriatric psychiatry: official journal of the American Association for Geriatric Psychiatry 2016;24:1084-1094. doi. org/10.1016/j.jagp.2016.06.005

23. Arts $\mathrm{MH}$ et al. Physical Frailty and Cognitive Functioning in Depressed Olde Adults: Findings From the NESDO Study. Journal of the American Medical Directors Association 2016;17:36-43. doi.org/10.1016/j.jamda.2015.07.016

24. Collard RM, Comijs HC, Naarding P, Oude Voshaar RC. Physical frailty: vulnerability of patients suffering from late-life depression. Aging \& mental health 2014;18:570 578. doi.org/10.1080/13607863.2013.827628

25. McAdams-DeMarco MA et al. Individual Frailty Components and Mortality in Kidney Transplant Recipients. Transplantation 2017;101:2126-2132. doi.org/10.1097/ TP.0000000000001546

26. Dent E, Hoogendijk, EO. Psychosocial factors modify the association of frailty with adverse outcomes: a prospective study of hospitalised older people. BMC geriatrics 2014;14:108. doi.org/10.1186/1471-2318-14-108

27. Collard RM, Arts M, Schene AH, Naarding P, Oude Voshaar RC, Comijs HC. The impact of frailty on depressive disorder in later life: Findings from the Netherlands Study of depression in older persons. European psychiatry: the journal of the Association of European Psychiatrists 2017;43:66-72. doi.org/10.1016/j. eurpsy.2017.01.003

28. Lugtenburg A et al. Subtypes of Late-Life Depression: A Data-Driven Approach on Cognitive Domains and Physical Frailty. The journals of gerontology. Series A Biological sciences and medical sciences 2021;76:141-150. doi.org/10.1093/gerona/ glaa110

29. Satake $\mathrm{S}$ et al. Prevalence of frailty among community-dwellers and outpatients in Japan as defined by the Japanese version of the Cardiovascular Health Study criteria Geriatrics \& Gerontology International 2017;17:2629-2934. doi: 10.1111/ggi.13129.

30. Aprahamian I et al. Depression is associated with self-rated frailty in older adults from an outpatient clinic: a prospective study. International psychogeriatrics 2019;31:425434. doi.org/10.1017/S104161021800100X

31. Kojima G, Iliffe S, Walters K. Frailty index as a predictor of mortality: a systematic review and meta-analysis. Age Ageing 2018;47:193-200. doi:10.1093/ageing/afx162

32. Borges MK et al. Could Frailty be an Explanatory Factor of the Association between Depression and Other Geriatric Syndromes in Later Life?. Clinical gerontologist 2020;1-11. doi.org/10.1080/07317115.2020.1836106

33. Borges $\mathrm{MK}$ et al. Depression as a determinant of frailty in late life. Aging \& mental health, 2020;1-7. doi.org/10.1080/13607863.2020.1857689

34. Aprahamian I et al. Design and protocol of the multimorbidity and mental health cohort study in frailty and aging (MiMiCS-FRAIL): unraveling the clinical and molecular associations between frailty, somatic disease burden and late life depression. BMC psychiatry 2020;20:573. doi.org/10.1186/s12888-020-02963-9

35. American Psychiatric Association (2013) Diagnostic and Statistical Manual of Mental Disorders: DSM-5, 5th ed. Arlington, USA

36. Sheikh JI, Yesavage JA. Geriatric Depression Scale (GDS): Recent evidence 
and development of a shorter version. Clinical Gerontologist 1986;5:165-172. doi:10.1300/J018v05n01_09

37. Kroenke K, Spitzer RL, Williams JB. The PHQ-9: validity of a brief depression severity measure. Journal of general internal medicine 2001;16:606-613. doi. org/10.1046/j.1525-1497.2001.016009606.x

38. Santos IS et al. Sensibilidade e especificidade do Patient Health Questionnaire-9 (PHQ-9) entre adultos da população geral [Sensitivity and specificity of the Patient Health Questionnaire-9 (PHQ-9) among adults from the general population]. Cadernos de saude publica 2013;29:1533-1543. doi.org/10.1590/0102-311x00144612

39. Morley JE et al. Frailty consensus: a call to action. Journal of the American Medical Directors Association 2013;14:392-397. doi.org/10.1016/j.jamda.2013.03.022

40. Aprahamian I et al. Feasibility and Factor Structure of the FRAIL Scale in Older Adults. Journal of the American Medical Directors Association 2017;18:367.e11-367. e18. doi.org/10.1016/j.jamda.2016.12.067

41. Lin SM et al. Comparison of 3 Frailty Instruments in a Geriatric Acute Care Setting in a Low-Middle Income Country. Journal of the American Medical Directors Association 2018;19:310-314.e3. doi:10.1016/j.jamda.2017.10.017

42. Mitnitski AB, Mogilner AJ, Rockwood K. Accumulation of deficits as a proxy measure of aging. The Scientific World Journal 2001;1:323-336. doi.org/10.1100/ tsw. 2001.58

43. Rockwood K, Andrew M, Mitnitski A. A comparison of two approaches to measuring frailty in elderly people. The journals of gerontology. Series A, Biological sciences and medical sciences, 2007;62:738-743. doi.org/10.1093/gerona/62.7.738

44. Rockwood K, Howlett SE. Age-related deficit accumulation and the diseases of ageing. Mechanisms of ageing and development 2019;180:107-116. doi.org/10.1016/j. $\operatorname{mad} .2019 .04 .005$

45. Clegg A et al. Development and validation of an electronic frailty index using routine primary care electronic health record data. Age and ageing 2016;45:353-360. doi org/10.1093/ageing/afw039

46. Oude Voshaar RC et al. Course of frailty stratified by physical and mental multimorbidity patterns: a five-year follow-up of 92,640 participants of the LifeLines cohort study. BMC Medicine 2021;19(1):29. doi.org/10.1186/s12916-021-01904-x

47. Apolinario D et al. Using temporal orientation, category fluency, and word recall for detecting cognitive impairment: the 10-point cognitive screener (10-CS). International journal of geriatric psychiatry 2016;31:4-12. doi.org/10.1002/gps.4282

48. Woods NF et al. Frailty: emergence and consequences in women aged 65 and older in the Women's Health Initiative Observational Study. Journal of the American Geriatrics Society 2005;53:1321-1330. doi.org/10.1111/j.1532-5415.2005.53405.x

49. Lakey SL et al. Antidepressant use, depressive symptoms, and incident frailty in women aged 65 and older from the Women's Health Initiative Observational Study. Journal of the American Geriatrics Society 2012;60:854-861. doi.org/10.1111/j.1532$5415.2012 .03940 . x$
50. Lohman M, Dumenci L, Mezuk B. Sex differences in the construct overlap of frailty and depression: evidence from the Health and Retirement Study. Journal of the American Geriatrics Society 2014;62:500-505. doi.org/10.1111/jgs.12689

51. Paulson D, Lichtenberg PA. Vascular depression: an early warning sign of frailty. Aging \& mental health 2013;17:85-93. doi.org/10.1080/13607863.2012.692767

52. Zhang $\mathrm{N}$ et al. Depressive symptoms are associated with incident frailty in a Chinese population: the Rugao Longevity and Aging Study. Aging clinical and experimental research, 2020;32:2297-2302. doi.org/10.1007/s40520-019-01409-x

53. Mayerl H, Stolz E, Freidl W. Frailty and depression: Reciprocal influences or common causes?. Social science \& medicine 2020;263:113273. https://doi.org/10.1016/j. socscimed.2020.113273

54. Chu XF et al. Frailty and incident depressive symptoms in a Chinese sample: the Rugao Longevity and Ageing Study. Psychogeriatrics : the official journal of the Japanese Psychogeriatric Society 2020;20:691-698. doi.org/10.1111/psyg.12565

55. Arts $\mathrm{MH}$ et al. Relationship between Physical Frailty and Low-Grade Inflammation in Late-Life Depression. Journal of the American Geriatrics Society 2015;63:1652-1657. doi.org/10.1111/jgs. 13528

56. Brown PJ et al. Inflammation, Depression, and Slow Gait: A High Mortality Phenotype in Later Life. The journals of gerontology. Series A, Biological sciences and medical sciences 2016;71:221-227. doi.org/10.1093/gerona/glv156

57. Arts, M. et al. Leucocyte telomere length is no molecular marker of physical frailty in late-life depression. Experimental gerontology, 2018;111:229-234. doi.org/10.1016/j. exger.2018.07.016

58. Melo RC et al. Prevalence of Frailty in Brazilian Older Adults: A Systematic Review and Meta-analysis. The journal of nutrition, health \& aging, 2020;24(7):708-716. doi org/10.1007/s12603-020-1398-0

59. Biella MM, Borges MK, Strauss J, Mauer S, Martinelli JE, Aprahamian I Subthreshold Depression Needs A Prime Time In Old Age Psychiatry? A Narrative Review Of Current Evidence. Neuropsychiatric disease and treatment 2019;15:27632772. doi.org/10.2147/NDT.S223640

60. Prince $\mathrm{M}$ et al. No health without mental health. Lancet 2007;370:859-877. doi. org/10.1016/S0140-6736(07)61238-0

How to cite this article: M.K. Borges, C.V. Romanini, N.A. Lima et al. Longitudinal Association between Late-Life Depression (LLD) and Frailty: Findings from a Prospective Cohort Study (MiMiCS-FRAIL). J Nutr Health Aging. 2021;25(7):895902; http://dx.doi.org/10.1007/s12603-021-1639-x 